\title{
SHORTER SPRINTS ELICIT GREATER CARDIORESPIRATORY AND MECHANICAL RESPONSES WITH LESS FATIGUE DURING TIME-MATCHED SPRINT INTERVAL TRAINING (SIT) SESSIONS
}

\author{
Stefano Benítez-Flores ${ }^{1}$, Arilson F.M. de Sousa ${ }^{1}$, Erick Carlos da Cunha Totón \\ Thiago Santos Rosa ${ }^{1}$, Sebastián Del Rosso ${ }^{1}$, Carl Foster ${ }^{2}$, and Daniel Boullosa ${ }^{1,3}$ \\ ${ }^{1}$ Catholic University of Brasilia, Águas Claras, DF, Brazil \\ ${ }^{2}$ University of Wisconsin La Crosse, La Crosse, WI, USA \\ ${ }^{3}$ College of Healthcare Sciences, James Cook University, Townsville, Australia
}

Original scientific paper

https://doi.org/10.26582/k.50.2.13

UDC: $796.422 .12: 612.766 .1$

\begin{abstract}
:
The aim of this study was to compare the physiological, mechanical and perceptual responses to two sprint interval training (SIT) sessions with very short vs. long sprints, and to verify if those differences could be reflected in measures of acute fatigue. Eleven physically active men performed, after the maximum oxygen consumption $\left(\mathrm{VO}_{2} \mathrm{max}\right)$ determination, SIT5s $(16 \times 5 \mathrm{~s}$ with $24 \mathrm{~s}$ of recovery $)$ and SIT20s $(4 \times 20 \mathrm{~s}$ with 120 s of recovery) in random order on a cycle ergometer. Physiological, mechanical, and perceptual responses were evaluated during and after the sessions. The countermovement jump (CMJ) height and autonomic control of heart rate (HR) were evaluated before and after the sessions. Diet was also controlled through recall questionnaires. During the training, SIT5s exhibited greater $\mathrm{HR}, \mathrm{VO}_{2}$, power output, and total work $(\mathrm{TW})(\mathrm{p}<.05)$. In contrast, respiratory exchange ratio (RER), rate of fatigue (RF), and blood lactate (BLa) \% accumulation were greater in SIT20s $(\mathrm{p}<.05)$. The OMNI-cycle Scale Rating of Perceived Exertion (OMNIcycle scale) and Feeling Scale (FS) scores were similar during both protocols ( $\mathrm{p}>.05)$. A faster HR recovery (HRR) and a higher CMJ height were observed after the SIT5s $(\mathrm{p}<.05)$. However, HR variability (HRV) was similarly depressed after both protocols ( $\mathrm{p}>.05)$. Some correlations between the mechanical and physiological responses were revealed only in the SIT5s. SIT5s was demonstrated to be more efficient as exhibited by greater mechanical responses associated with a higher aerobic activity, when compared to the volume-matched SIT protocol of longer sprints. Simple monitoring tools such as HRR and CMJ could help practitioners to detect differences in acute fatigue after different SIT sessions.
\end{abstract}

Key words: high intensity interval training, mechanical responses, physiological responses, perceptual responses, acute fatigue

\section{Introduction}

Lack of time is one of the main arguments expressed for not practicing regular physical activity (PA) (Trost, Owen, Bauman, Sallis, \& Brown, 2002). This problem could be solved with the implementation of interval training, a highly effective training modality that requires less time commitment and has multiple potential health benefits (Batacan, Duncan, Dalbo, Tucker, \& Fenning, 2017). Interval training can be defined as an intermittent exercise that involves alternating short periods of high-intensity activity with periods of lower-intensity activity for recovery (Batacan, et al., 2017). Interval training can be classified into two categories: high-intensity interval training (HIIT), which typically denotes submaximal efforts eliciting $\geq 80 \%$ of maximal heart rate (HR), and sprint interval training (SIT), which involves "all out" efforts, generally requiring very high levels of power output (Gibala \& Hawley, 2017). SIT has been shown to be a timeefficient strategy to promote metabolic adaptations in skeletal muscles (Gibala, Little, MacDonald, \& Hawley, 2012), while improving maximum oxygen consumption $\left(\mathrm{VO}_{2} \max \right)$ and power output (Sloth, Sloth, Overgaard, \& Dalgas, 2013) with a comparatively small training workload.

Although clearly developed as an experimental model of muscular adaptation, the applicability of classical SIT protocol (4-6 sprints $\times 30$ s) for unhe- 
althy and/or sedentary individuals has been questioned (Gaesser \& Angadi,2011), because it is extremely demanding and difficult (Gibala, et al., 2012; Verney, Duclos, \& Thivel, 2017). Accordingly, most studies have included highly motivated young active adults (Skleryk, et al., 2013). Moreover, after the completion of SIT protocols, there have been reports of faints, respiratory events, nausea, lightheadedness, and vomiting (Verney, Duclos, \& Thivel, 2017). Thus, the adherence to SIT has been questioned in literature (Hardcastle, Ray, Beale, \& Hagger, 2014).

The reduction of work-interval duration could be one of the best strategies to facilitate SIT application (Zelt, et al., 2014). Previous studies have suggested that shorter sprints (e.g., 10-20s) were equally effective for improving $\mathrm{VO}_{2} \max$, lactate threshold, critical power, time to exhaustion, time trial performance, anaerobic power, and repeated sprint performance (Hazell, MacPherson, Gravelle, \& Lemon, 2010; Yamagishi \& Babraj, 2017; Zelt, et al., 2014) as the classical SIT, while being better tolerated by participants and allowing greater power outputs (Hazell, et al., 2010; Yamagishi \& Babraj, 2017). Furthermore, a recent review by Vollaard and Metcalfe (2017) highlights the effectiveness of reducing sprint duration and repetitions for health benefits. The similar improvements with modified SIT protocols may be due to the effects of brief maximal power efforts which seem to be the key stimulus for physiological adaptations (Hazell, et al., 2010, Zelt, et al., 2014). Peak power (PP) output is a mechanical variable that is generally achieved during the first 5s (Micklewright, Alkhatib, \& Beneke, 2006), therefore its achievement could be more important for physiological adaptations than its maintenance (Hazell, et al., 2010).

Recently, Islam, Townsend, and Hazell (2017) showed that a protocol with very short sprints (i.e., 5s) elicited higher cardiorespiratory responses and energy expenditure (EE) without compromising post-exercise EE, compared to longer sprints (i.e., 15 or $30 \mathrm{~s})$ with the matched training volume (30 min). Additionally, and critically, very short sprints were more enjoyable and led to greater intentions to continue SIT (Townsend, et al., 2017). However, the physiological impact associated with acute fatigue and recovery using different modified SIT protocols has not been described. In this respect, practical and simple evaluation tools such as HR recovery (HRR), HR variability (HRV) (Stanley, Peake, \& Buchheit, 2013), and vertical jump performance (Jimenez-Reyes, et al., 2016; Morcillo, et al., 2015) could be useful for identifying differences in acute fatigue between SIT protocols. This information could be important for designing complex training sessions in which strength exercises are performed after endurance efforts (Fyfe, Bartlett, Hanson, Stepto, \& Bishop, 2016). Furthermore, given that most previous studies did not equate training workloads, it would be also very important to describe the relationship between external (i.e., mechanical parameters) and internal load (i.e., physiological responses) during SIT protocols for a better understanding of the impact of mechanical parameters on acute physiological responses.

Thus, the aims of the current study were to compare physiological, mechanical, and perceptual acute responses between two different SIT's protocols of very short vs. long efforts but with the matched volume [SIT with $5 \mathrm{~s}$ efforts (SIT5s) vs. SIT with 20s efforts (SIT20s)], and to verify if the differences in external and internal workloads would influence acute fatigue parameters (i.e., HRR, HRV and jump performance). Our hypothesis was that participants in the SIT5s would reach higher mechanical and metabolic responses with lower perceptual scores, favoring a lower acute fatigue and a better recovery.

\section{Methods}

\section{Participants}

Eleven healthy active young men participated in this study. The participants' characteristics are presented in Table 1. All partipants practiced recreational sports (e.g., basketball, jiu-jitsu, running, soccer, strength training, swimming and triathlon) $\geq 3$ times/wk during $\geq 60 \mathrm{~min}$, but none were highlevel competitive athletes. Inclusion criteria were: (1) being very physically active according to the International Physical Activity Questionnaire (IPAQ); (2) not consuming any type of nutritional supplement or tobacco product; (3) to be free of any orthopedic injury that could influence test performances. Participants were instructed to refrain from PA and alcohol consumption for $48 \mathrm{~h}$ before all sessions and also to avoid caffeine beverages in the morning of evaluations. They were asked not to change daily life habits (work, sleep, etc.). Prior to the participation, the experimental procedures and potential risks were explained to all participants in written and verbal forms. Thereafter, they provided written informed consents. This study was approved by the Ethics Committee of Catholic University of Brasilia (protocol number CAAE 54813016.0.0000.0029).

Table 1. Participants' characteristics

\begin{tabular}{lc}
\hline & $(\mathrm{M} \pm \mathrm{SD})$ \\
\hline Age (years) & $26.2 \pm 3.6$ \\
Body height $(\mathrm{cm})$ & $176.2 \pm 0.8$ \\
Body weight $(\mathrm{kg})$ & $75.1 \pm 8.2$ \\
BMI $\left(\mathrm{kg} \cdot \mathrm{m}^{-2}\right)$ & $24.1 \pm 1.2$ \\
Body fat $(\%)$ & $10.9 \pm 3.3$ \\
Systolic blood pressure $(\mathrm{mmHg})$ & $110.9 \pm 9.4$ \\
Diastolic blood pressure $(\mathrm{mmHg})$ & $78.1 \pm 7.5$ \\
\hline
\end{tabular}

Note. BMI=body mass index. 


\section{Study design}

Participants completed, in a randomized crossover design, three exercise sessions of one hour separated by $48 \mathrm{~h}$, on an electronically braked cycle ergometer (Excalibur Sport V 2.0, Lode, Groningen, Netherlands), with the last two sessions in randomized order. During the first session, after arrival to the laboratory, participants completed the IPAQ. Nutrition recalls were also given to participants. The following anthropometric measures were collected: body mass $(\mathrm{kg})$ using a digital balance (Toledo, Toledo2286PP,São Paulo, Brazil), stature (m) using a stadiometer (Stadi-O-Meter,NovelProductsInc, Rockton, Illinois, USA), and body composition by dual energy X-ray absorptiometry (DEXA) (Lunar DPX-IQ, Wisconsin, USA). Subsequently they performed 2-4 countermovement jumps (CMJ) for the familiarization purposes. Immediately after these procedures, adjustments in cycle ergometer were made for each participant. These adjustments were registered and subsequently used during all exercise sessions. For the $\mathrm{VO}_{2}$ max determination, an incremental test was performed during which mechanical and physiological data were collected from participants. Ten minutes after the $\mathrm{VO}_{2} \max$ test, a familiarization with the SIT protocols was carried out allowing the participants to perform 1-2 all-out sprints of 5 and 20s (Gillen, et al., 2014). During the second and third session, two volumeequated SIT protocols (SIT5s and SIT20s) were performed in randomized order. For both protocols, nutrition was controlled. Physiological, mechanical and perceptual data were collected pre-, during and post-exercise (see Figure 1). All sessions were performed on the same days of the week (Monday, Wednesday and Friday), at the same time (8-11a.m.), and under constant ambient conditions (temperature $=21-23^{\circ} \mathrm{C}$, relative humidity $=75-85 \%$ ). In every session, prior to testing, the gas analyzer was cali- brated with gases of known concentrations and a 3 -L syringe following manufacturer instructions.

\section{Incremental test}

Participants were asked not to eat $3 \mathrm{~h}$ before the test and to maintain adequate hydration. The protocol was used in previous studies (Gillen, et al., 2014) and started with a warm-up of one minute at $50 \mathrm{~W}$. Subsequently, progressive increases of $1 \mathrm{~W}$ every 2 s (i.e., $30 \mathrm{~W}$ per minute ramp slope) were performed until exhaustion or when the pedalling cadence fell below 50rpm. Participants were verbally encouraged to exercise to volitional exhaustion. Oxygen consumption $\left(\mathrm{VO}_{2}\right)$, carbon dioxide production $\left(\mathrm{VCO}_{2}\right)$ and ventilation $\left(\mathrm{V}_{\mathrm{E}}\right)$ were measured every $20 \mathrm{~s}$ using a metabolic cart (Cortex, Metalyzer, Leipzig, Germany). The $\mathrm{VO}_{2}$ max was defined as the highest $\mathrm{VO}_{2}$ value registered during a 20 s period and was confirmed when a minimum of two of the following criteria were met: (1) a respiratory exchange ratio (RER) higher than 1.2 ; (2) peak HR $\geq 90 \%$ of the age predicted maximum (i.e., 220 minus age);or (3) visible exhaustion. The coefficient of variation for the $\mathrm{VO}_{2} \mathrm{max}$ determination in our laboratory is $<10 \%$. Maximum power $\left(\mathrm{P}_{\max }\right)$ and maximum $\mathrm{HR}\left(\mathrm{HR}_{\max }\right)$ were determined during a 20 s period at the end of the test.

\section{SIT sessions}

On the second and third sessions, participants performed SIT5s $(16 \times 5 \mathrm{~s}$ followed by $24 \mathrm{~s}$ of active recovery) or SIT20s $(4 \times 20$ s followed by 120 s of active recovery) in randomized order. The protocols were volume-matched $[7 \mathrm{~min} 20 \mathrm{~s}$ of exercise ( $1 \mathrm{~min} 20 \mathrm{~s}$ of sprints and $6 \mathrm{~min}$ recovery), and $12 \mathrm{~min}$ 20 s for total time [7min 20s of training ( $2 \mathrm{~min}$ of warming up, and $3 \mathrm{~min}$ of cooling down)]. The torque factor was set to $0.7 \mathrm{~N} \cdot \mathrm{m}$ as is typical for normal healthy test subjects (Wingate for Windows

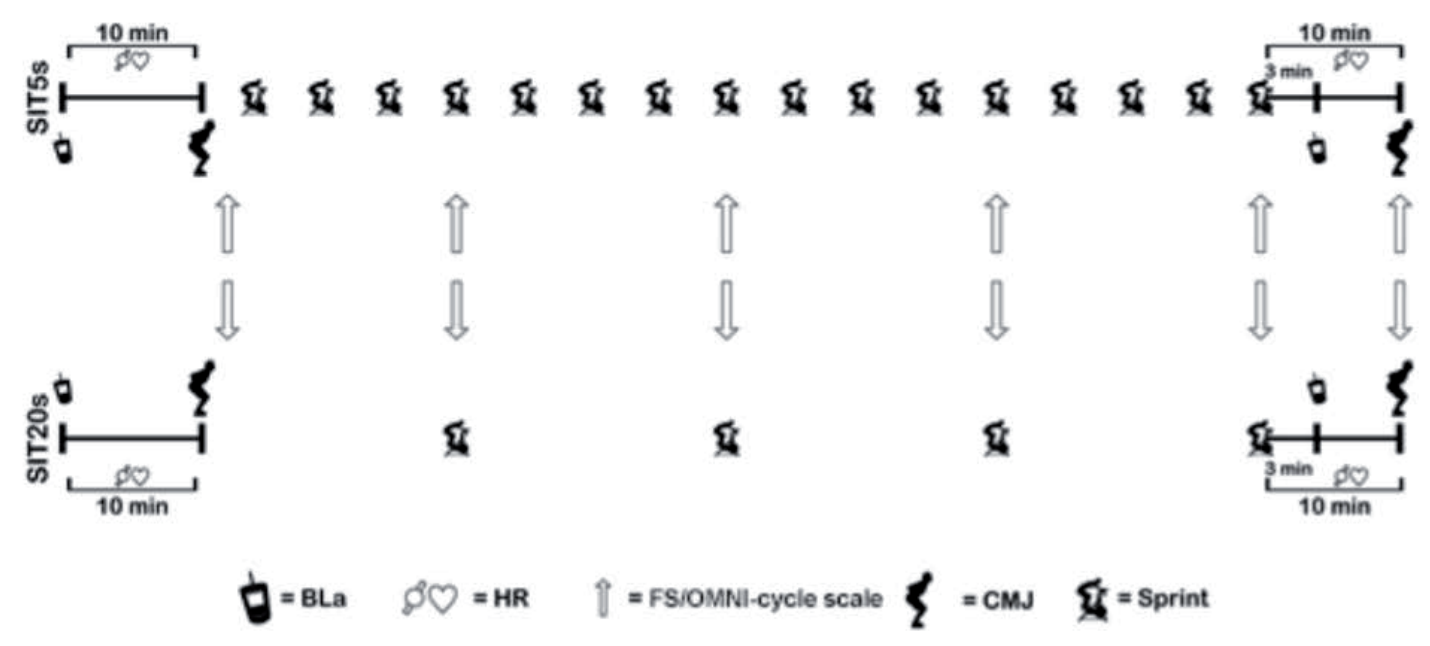

Figure 1. BLa=blood lactate, $H R=$ heart rate, FS=feeling scale, OMNI-cycle scale=OMNI-cycle scale rating of perceived exertion, $C M J=$ countermovement jump, SIT5s=sprint interval training with 5 s efforts, SIT20s=sprint interval training with 20 s efforts. 
software version 1, Lode BV). Warm-up, recovery and cooling down were performed at a cadence of $50 \mathrm{rpm}$ and with a load of 50W. All sprints were performed in a seated position. Participants were asked to perform at their maximum for each interval with the main investigator providing strong verbal encouragement. Two seconds before the load was applied, participants were required to reach the highest pedalling frequency possible and to maintain this frequency until the end of each interval.

\section{Physiological variables}

During the two SIT protocols, $\mathrm{HR}, \mathrm{VO}_{2}$, and RER were continuously monitored and averaged over 20s periods (Cortex, Metalyzer, Leipzig, Germany). The EE per minute was calculated assuming $5 \mathrm{kcal}$ per $\mathrm{L}$ of $\mathrm{O}_{2}$ consumed (Williams, et al., 2013). Blood lactate (BLa) concentration was determined at rest and $3 \mathrm{~min}$ after the end of SIT protocols with a portable lactate analyzer (Accutrend, Roche Diagnostics, Mannheim, Germany).

\section{Mechanical variables}

Absolute and relative PP, minimum power (MIP), mean power (MEP), total work (TW), work during the first sprint $\left(\mathrm{W}_{\text {first }}\right)$, rate of fatigue $(\mathrm{RF})$, and maximal pedaling rate $\left(\mathrm{RPM}_{\max }\right)$ were recorded during each effort using a cycle ergometer in Wingate mode (Lode BV, Groningen, The Netherlands). Mechanical variables were defined as follows: PP as the highest single value of power output, MIP as the lowest single value of power output, MEP as the average power output. TW was obtained by multiplying MEP by the duration of the protocol, whereas RF was calculated as the degree of power drop-off during the effort calculated as a percentage of peak power, and $\mathrm{RPM}_{\max }$ as the maximum number of revolutions per minute achieved. The average of PP, MIP, MEP, RF and $\mathrm{RPM}_{\max }$, and the sum of the work in all efforts (i.e., TW) in both sessions were subsequently calculated.

\section{Perceptual variables}

To determine perceived exertion, the OMNIcycle Scale Rating of Perceived Exertion (OMNIcycle scale) (Robertson, et al., 2004) was used. This scale was validated for adults for RPE during cycle exercise, being of easier application than the classic Borg scale (6-20) (Robertson, et al., 2004). The Feeling Scale (FS) was used to measure the affective valence (pleasure and displeasure), ranging from -5 (very bad) to +5 (very good) (Hardy \& Rejeski, 1989). Both scales were applied pre-exercise, during SIT protocols after sprints 4, 8, 12, 16 for SIT5s, and after each sprint for SIT20s, and $10 \mathrm{~min}$ after both protocols.

\section{HR measures}

HR was measured pre- and post- each protocol during $10 \mathrm{~min}$. The last $5 \mathrm{~min}$ of every recording were used for HRV analyses. The first $5 \mathrm{~min}$ of recovery after the last effort of each protocol were used for HRR analyses. Data collection was carried out with participants pedaling at a steady cadence of 50rpm with a load of 50W. The HR was recorded using a monitor with an accuracy of $1 \mathrm{~ms}$ (RS800CX, Polar Electro Oy, Kempele, Finland). Subsequently, data were filtered with specific software (Polar Pro-Trainer 5 version 5.40.170, Polar Electro Oy, Kempele, Finland). The HRV was analyzed with custom designed software (Kubios HRV Analysis version 2.2, The Biomedical Signals Analysis Group, University of Kuopio, Finland). The variables selected for the analyses were: standard deviation of R-R intervals (SDNN), root mean square of successive differences in R-R interval (RMSSD), short-term beat-to-beat R-R variability from the Poincare plot (SD1), long-term beat-to-beat variability from the Poincaré plot (SD2), sample entropy (SampEn), detrended fluctuations of shortterm fractal scaling $(\alpha 1)$, and correlation dimension (D2) (Boullosa, Barros, Del Rosso, Nakamura, \& Leicht, 2014; Weippert, Behrens, Rieger, Kumar, \& Behrens, 2015). Relative HRR was defined as the difference between $\mathrm{HR}$ registered at the end of exercise (HRend) and after $30 \mathrm{~s}, 1 \mathrm{~min}, 2 \mathrm{~min}, 3 \mathrm{~min}$ and $5 \mathrm{~min}$ (i.e., $\Delta 30 \mathrm{~s}, \Delta 1 \mathrm{~min}, \Delta 2 \mathrm{~min}, \Delta 3 \mathrm{~min}$ and $\Delta 5 \mathrm{~min}$ ) (Boullosa, et al.,2014).

\section{Vertical jump}

CMJ height was recorded using a contact platform connected to a digital timer through an interface (ChronoJump 1.6.2, BoscoSystem, Spain). The flight time of each jump was recorded and converted automatically to jump height with the specific software that was connected to a computer. $\mathrm{CMJ}$ height was measured pre- and $10 \mathrm{~min}$ after each SIT session. Two CMJs were performed at each moment and the resulting average was used for futher analyses (Claudino, et al., 2016).

\section{Dietary control}

Dietary consumption for $24 \mathrm{~h}$ was only monitored before the first training session and we requested participants to replicate the same dietary intake $24 \mathrm{~h}$ before the second training session. A dietician instructed participants how to complete the diet recall questionnaires. All diet recalls were analyzed by the same dietician for total kilocalories, protein, carbohydrate, fat, vitamin $\mathrm{C}$, vitamin $\mathrm{E}$, and vitamin A intake using a custom software (Smart data, Sao Paulo, Brazil). 


\section{Statistical analyses}

Data are presented as $\mathrm{M} \pm \mathrm{SD}$. Normality was assessed by means of standard distribution measures (i.e.skewness and kurtosis) visual inspection of Q-Q plots and box plots, and the ShapiroWilk test. Variables with a non-normal distribution were log-transformed (Ln) for analysis. Comparison of selected variables between SIT protocols were carried out using $t$-tests for paired samples. Pearson product-moment correlation coefficient ( $r$ ) was used to assess the relationships between selected variables. An analysis of variance (ANOVA) for repeated measures was used with time effect (before and after training) and training protocol (SIT5S vs. SIT20S) for the evaluation of selected variables before, during and after the sessions. Mauchly's sphericity was tested and if sphericity could not be assumed, then the Greenhouse-Geisser correction was used. Cohen's d was calculated for effect size (ES) analyses. All statistics were performed with appropriate software IBM SPSS Statistics for Windows ${ }^{\circledR}$ (Version 20.0, Armonk, NY, USA). The alpha level was set at a $\mathrm{p}<.05$. All graphics were made with GraphPad Prism 6.01 (GraphpadSoftware, San Diego, CA, USA).

\section{Results}

During the incremental test, participants achieved a $\mathrm{VO}_{2} \max$ of $45.9 \pm 3.7 \mathrm{~mL} \cdot \mathrm{kg}^{-1} \cdot \mathrm{min}^{-1}$, with a $\mathrm{P}_{\max }$ of $322.3 \pm 14.4 \mathrm{~W}$, and a $\mathrm{HR}_{\max }$ of $173 \pm 11$ beat $\cdot \min ^{-1}$.

\section{Physiological responses}

MeanHR, HRpeak and \%HRmax were higher in SIT5s $(\mathrm{p}<.05)$. MeanVO, $\mathrm{VO}_{2}$ peak and $\% \mathrm{VO}_{2} \max$ were higher in SIT5s $(\mathrm{p}<.001)$. MeanRER was greater in SIT20s $(\mathrm{p}<.001)$. In contrast, EE was greater in SIT5s $(\mathrm{p}<.001)$. Significant $(\mathrm{p}<.05)$ protocol, time, and protocol $\times$ time interaction effects were found for BLa. Thus, when $\mathrm{BLa} \%$ changes (pre- to post-exercise) were compared between the protocols, a significant difference was found between the sessions: $[2.3 \pm 0.6$ to $14.1 \pm 2.5(+11.7)$ vs. $1.9 \pm 0.2$ to $16.1 \pm 2.1(+14.2 \%)$ for SIT5s and SIT20s, respectively $(\mathrm{p}=.006)]$ (see Figure 2).

\section{Mechanical variables}

PP (W) was similar between SIT sessions ( $p>05$ ). In contrast, MEP (W), MIP (W) and TW $(\mathrm{kJ})$ were higher in SIT5s ( $\mathrm{p}<.001)$. However, RF(\%) and $\mathrm{TW}_{\text {first }}(\mathrm{kJ})$ were greater in SIT20s $(\mathrm{p}<.001)$.

Table 2. Physiological comparison between SIT protocols

\begin{tabular}{|c|c|c|c|c|}
\hline & SIT5s & SIT20s & $\mathrm{p}$ & ES \\
\hline MeanHR (beat·min-1) & $156 \pm 10$ & $141 \pm 11$ & .00 & .55 \\
\hline HRpeak (beat:min-1 ${ }^{1}$ ) & $170 \pm 9$ & $166 \pm 9$ & .04 & .21 \\
\hline$\%$ HRmax (beat $\cdot \min ^{-1}$ ) & $90.1 \pm 5.7$ & $81.5 \pm 4.8$ & .00 & .58 \\
\hline $\mathrm{MeanVO}_{2}\left(\mathrm{~mL} \cdot \mathrm{kg}^{-1} \cdot \mathrm{min}^{-1}\right)$ & $37.1 \pm 3.1$ & $27.1 \pm 1.3$ & .00 & .90 \\
\hline $\mathrm{VO}_{2}$ peak $\left(\mathrm{mL} \cdot \mathrm{kg}^{-1} \cdot \mathrm{min}^{-1}\right)$ & $43.5 \pm 3.3$ & $40.8 \pm 3.4$ & .00 & .37 \\
\hline 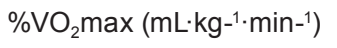 & $80.8 \pm 5.3$ & $59 \pm 4.3$ & .00 & .91 \\
\hline MeanRER & $1.20 \pm 0.06$ & $1.44 \pm 0.08$ & .00 & .86 \\
\hline EE (kcal) & $101.7 \pm 8.9$ & $74.9 \pm 8.4$ & .00 & .84 \\
\hline
\end{tabular}

Note. Data are $\mathrm{M} \pm \mathrm{SD}$. $\mathrm{HR}=$ heart rate, $\mathrm{VO}_{2} \mathrm{max}=$ maximum oxygen consumption, $\mathrm{RER}=$ respiratory exchange ratio, $\mathrm{EE}=\mathrm{energy}$ expenditure, $\mathrm{kcal}=\mathrm{kilocalories,} \mathrm{ES}=$ effect size.

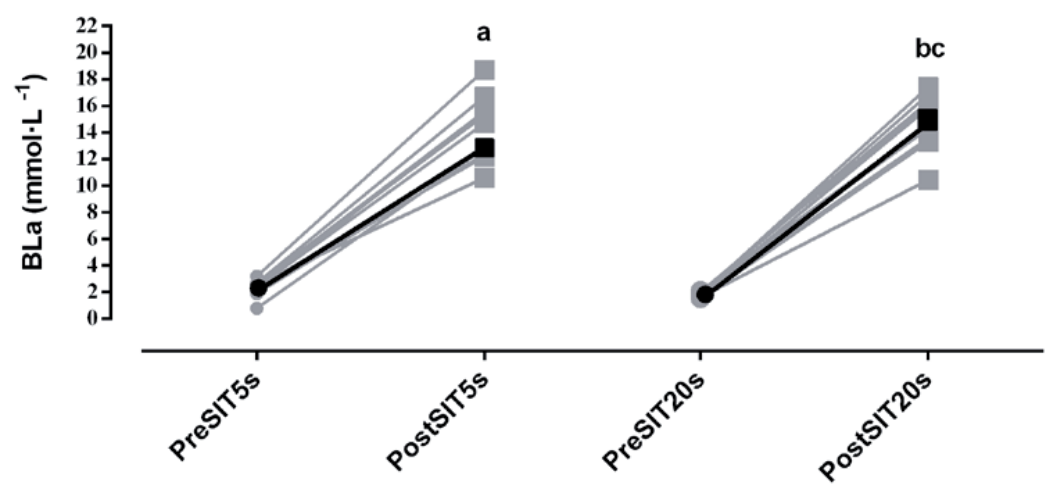

Figure 2. Individual and group pre-and post-blood lactate (BLa) concentrations in both training sessions. Thin lines are individual data and the thick lines are means. Effect of time ( $p<.01)$, effect of group $(p=.025)$ and effect of interaction ( $p=.004) ;{ }^{a} v \mathrm{vs}$. pre-SIT5s; ${ }^{b}$ vs. post-SIT5s; ${ }^{c}$ vs. pre-SIT20s. 


\section{Perceptual variables}

There were no significant differences between the protocols before, during and after exercise for OMNI-cycle scale ( $p>05)$ and the FS ( $p>05)$ at any time point (see Figure 3).

\section{HR measures}

The significant differences were found in all HRV parameters except for $\alpha 1$ (see Table 4 ) between pre- to post- values in both sessions $(\mathrm{p}<.001)$. However, there were no differences between the sessions. On the other hand, for HRR, the significant differences were found for $\Delta 30 \mathrm{~s}$ (beat $\mathrm{min}^{-1}$ ) after SIT5s $(\mathrm{p}<.05)$ with a tendency $(\mathrm{p}<.1$; ES: .44-.46) detected for $\Delta 1-3 \mathrm{~min}$ (see Table 5).

\section{Vertical jump}

The significant $(\mathrm{p}<.05)$ group, time, and group $x$ time interaction effects were identified for CMJ performance when relative pre- to post-exercise values were compared between the conditions $[34.3 \pm 4.6$ to $33.9 \pm 3.2(-0.45 \%)$ vs. $34.9 \pm 5.8$ to $30.7 \pm 3.6(-4.19 \%)$ for SIT5s and SIT20s, respectively $(\mathrm{p}=.019)]$ (see Figure 4).

\section{Dietary control}

No significant differences $(p>05)$ were noted in dietary intake over the $24 \mathrm{~h}$ period prior to each SIT protocol: $2630 \pm 733$ vs. $2655 \pm 950$ kcals; $126 \pm 40$ vs. $140 \pm 58 \mathrm{~g}$ of protein; $345 \pm 132 \mathrm{vs.} 318 \pm 115 \mathrm{~g}$ of carbohydrate; $84 \pm 42$ vs. $87 \pm 66 \mathrm{~g}$ of fat; $108 \pm 103$

Table 3. Mechanical comparison between SIT protocols

\begin{tabular}{lccll}
\hline & SIT5s & SIT20s & $p$ & ES \\
\hline PP $(\mathrm{W})$ & $922.8 \pm 84.9$ & $910.1 \pm 107.7$ & .56 & .06 \\
PP $\left(\mathrm{W} \cdot \mathrm{kg}^{-1}\right)$ & $12.3 \pm 0.8$ & $12.1 \pm 0.7$ & .50 & .13 \\
$\mathrm{MEP}(\mathrm{W})$ & $754.5 \pm 85.2$ & $608.8 \pm 71.3$ & .00 & .68 \\
$\mathrm{MIP}(\mathrm{W})$ & $575.3 \pm 89.2$ & $426.3 \pm 61.6$ & .00 & .70 \\
$\mathrm{~W}_{\text {first }}(\mathrm{kJ})$ & $4.5 \pm 0.6$ & $14.27 \pm 1.9$ & .00 & .96 \\
TW $(\mathrm{kJ})$ & $58.1 \pm 6.6$ & $48.4 \pm 5.6$ & .00 & .62 \\
TW $\left(\mathrm{J} \cdot \mathrm{kg}^{-1}\right)$ & $776.5 \pm 74$ & $645.3 \pm 49.9$ & .00 & .72 \\
$\mathrm{RPM}$ & $138.6 \pm 7.9$ & $133.9 \pm 8.6$ & .08 & .27 \\
$\mathrm{RF}(\%)$ & $37.9 \pm 8.1$ & $53.5 \pm 5.7$ & .00 & .74 \\
\hline
\end{tabular}

Note. Data are $M \pm S D$. $P P=$ peak power, $M E P=$ mean power, $M I P=$ minimum power, $W_{\text {first }}=$ work during the first sprint, $T W=$ total work, $\mathrm{RPM}_{\text {max }}=$ maximal pedaling rate, $\mathrm{RF}=$ rate of fatigue, $\mathrm{W}=$ watts, $\mathrm{kJ}=$ Kilojoules, $\mathrm{ES}=$ effect size.

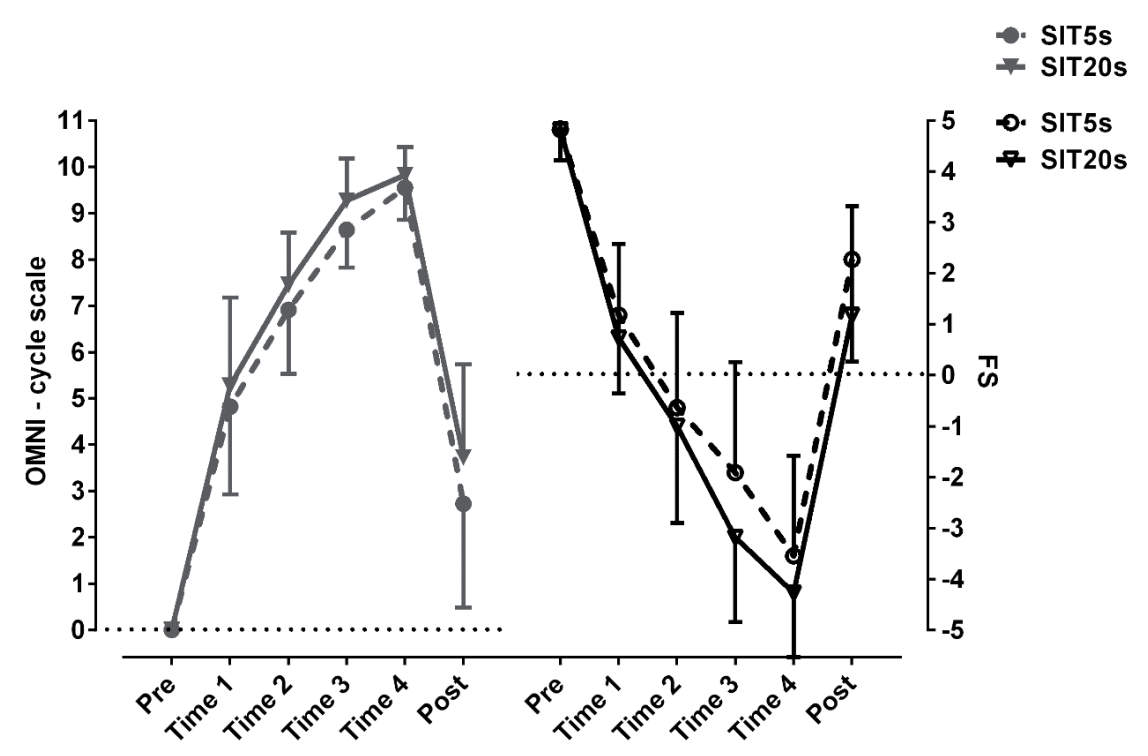

Figure 3. Differences between scales before, during and after the training sessions. OMNI-cycle scale=OMNI-cycle scale rating of perceived exertion, $F S=$ feeling scale. 
Table 4. Comparison of HRV measures before and after both SIT protocols

\begin{tabular}{lcccccc}
\hline & \multicolumn{2}{c}{ SIT5s } & \multicolumn{2}{c}{ SIT20s } & p & ES \\
\cline { 2 - 7 } & Pre & Post & Pre & Post & \\
\hline SDNN (ms) & $36.02 \pm 14.04$ & $14.38 \pm 3.47$ & $42.50 \pm 18.32$ & $11.66 \pm 3.70$ & .00 & .75 \\
RMSSD (ms) & $24.9 \pm 15.18$ & $4.82 \pm 2.17$ & $28.64 \pm 19.36$ & $5.28 \pm 2.76$ & .00 & .68 \\
SD1 (ms) & $17.63 \pm 10.75$ & $3.42 \pm 1.53$ & $20.28 \pm 13.73$ & $3.72 \pm 1.94$ & .00 & .67 \\
SD2 (ms) & $47.38 \pm 17.64$ & $19.93 \pm 4.99$ & $56.24 \pm 23.04$ & $15.98 \pm 5.00$ & .00 & .75 \\
SampEn & $1.43 \pm 0.25$ & $0.98 \pm 0.39$ & $1.31 \pm 0.26$ & $1.04 \pm 0.33$ & .00 & .43 \\
a1 & $1.31 \pm 0.26$ & $1.29 \pm 0.24$ & $1.31 \pm 0.27$ & $1.11 \pm 0.16$ & .09 & .15 \\
D2 & $1.85 \pm 1.62$ & $0.12 \pm 0.19$ & $2.11 \pm 1.65$ & $0.04 \pm 0.06$ & .00 & .59 \\
\hline
\end{tabular}

Note. Data are $M \pm S D$. SDNN=standard deviation of R-R intervals, RMSSD=root mean square of the successive differences in $R-R$ interval, SD1=short-term beat-to-beat $R-R$ variability from the Poincaré plot, SD2=long-term beat-to-beat variability from the Poincaré plot, SampEn=sample entropy, $\alpha 1=$ detrended fluctuations of short-term fractal scaling, D2=correlation dimension, $p=$ time effect, ES=effect size.

Table 5. Comparison of HRR measures before and after both SIT protocols

\begin{tabular}{|c|c|c|c|c|}
\hline & SIT5s & SIT20s & $p$ & ES \\
\hline HRend (beat: $\mathrm{min}^{-1}$ ) & $169 \pm 10$ & $160 \pm 9$ & .42 & .42 \\
\hline$\Delta 30$ s $\left(\right.$ beat $\cdot \mathrm{min}^{-1}$ ) & $18 \pm 5$ & $10 \pm 5$ & .02 & .62 \\
\hline$\Delta 1 \min \left(\right.$ beat $\left.\cdot \mathrm{min}^{-1}\right)$ & $29 \pm 9$ & $20 \pm 8$ & .07 & .46 \\
\hline$\Delta 2 \min \left(\right.$ beat $\left.\cdot \mathrm{min}^{-1}\right)$ & $41 \pm 8$ & $32 \pm 10$ & .08 & .44 \\
\hline$\Delta 3 \min \left(\right.$ beat $\left.\cdot \mathrm{min}^{-1}\right)$ & $45 \pm 7$ & $36 \pm 10$ & .09 & .46 \\
\hline$\Delta 5 \min \left(\right.$ beat $\left.\cdot \mathrm{min}^{-1}\right)$ & $47 \pm 10$ & $46 \pm 9$ & .85 & .05 \\
\hline
\end{tabular}

Note. Data are M $\pm S D$. HRend=heart rate at the end of the sessions, $\Delta 30$ seg-5min=difference between HRend and $H R$ at $30 \mathrm{~s}, 1 \mathrm{~min}$, $2 \mathrm{~min}, 3 \mathrm{~min}$ and $5 \mathrm{~min}$ post-test, respectively. ES=effect size.

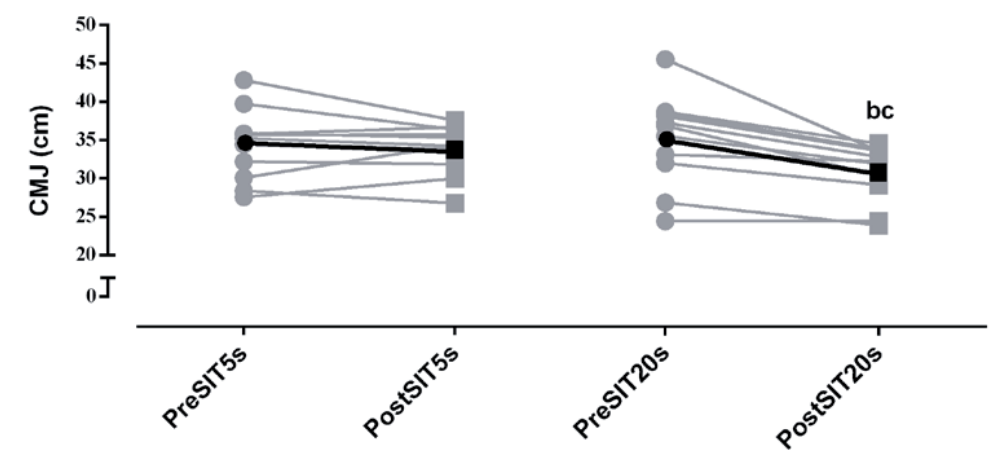

Figure 4. Individual and group pre- to post-countermovement jump (CMJ) heights in both training sessions. Thin lines are individual data and the thick lines are means. Effect of time $(p=.015)$, effect of group $(p=.018)$ and effect of interaction $(p<.01)$; ${ }^{b}$ vs. post-SIT5s; ${ }^{c}$ vs. pre-SIT20s.

vs. $137 \pm 131 \mathrm{mg}$ of vitamin $\mathrm{C} ; 9 \pm 11$ vs. $9 \pm 9 \mathrm{mg}$ of vitamin E; $427 \pm 525$ vs. $415 \pm 612 \mu \mathrm{g}$ of vitamin A; for SIT5s vs. SIT20s, respectively.

\section{Relationships between external and internal workload parameters}

\section{SIT5s}

There was a significant correlation between absolute PP and EE $(r=.79 ; p=.004)$. There were significant correlations between absolute MEP with
meanRER $(\mathrm{r}=-.69 ; \mathrm{p}=.017)$ and $\mathrm{EE}(\mathrm{r}=.63 ; \mathrm{p}=.037)$. There were significant correlations between TW with meanRER $(\mathrm{r}=-.70 ; \mathrm{p}=.016)$ and $\mathrm{EE}(\mathrm{r}=.62$; $\mathrm{p}=.045)$. There was a significant correlations between $\mathrm{W}_{\text {first }}$ and $\mathrm{EE}(\mathrm{r}=.68 ; \mathrm{p}=.019)$.

\section{SIT20s}

There were no significant correlations between mechanical and physiological variables during this session. A significant correlation between $\triangle \mathrm{CMJ}$ and meanRER $(\mathrm{r}=-.71 ; \mathrm{p}=.014)$ was revealed. 


\section{Discussion and conclusions}

The main findings of the current study were that the SIT protocol with very short sprints (SIT5s) elicited greater cardiorespiratory $\left(\mathrm{HR}\right.$ and $\left.\mathrm{VO}_{2}\right)$ and mechanical responses (MEP, MIP and TW), lower glycolytic activity (RER and BLa), less neuromuscular fatigue (RF and $\mathrm{CMJ}$ ) and a faster cardiac parasympathetic reactivation (HRR) compared to the SIT session of equated volume but with longer sprints (SIT20s). However, there were no differences between protocols for perceptual and affective responses during and after the sessions. Additionally, some correlations were found between mechanical and physiological responses in SIT5s, but not in SIT20s. These results are partially in agreement with recent findings observed in sprint running (Islam, et al., 2017) and provide further support to the appropriateness of SIT sessions with shorter efforts (Vollaard \& Metcalfe, 2017).

Following the findings of the recent study by Islam, et al., (2017) who reported higher fat oxidation rates when applying very shorts bouts (i.e., $5 \mathrm{~s}$ ), the current study demonstrated a greater mean HR, $\mathrm{VO}_{2}$ and hence a greater EE during SIT5s when compared to SIT20s. These results are not surprising as $\mathrm{VO}_{2} \max$ (McGawley \& Bishop, 2015; Tomlin \& Wenger, 2002), muscle $\mathrm{O}_{2}$ availability (Balsom, Gaitanos, Ekblom, \& Sjödin, 1994; Billaut \& Buchheit, 2013), and $\mathrm{O}_{2}$ kinetics (Dupont, Millet, Guinhouya, \& Berthoin, 2005) have demonstrated to be essential factors for ATP regeneration via phosphocreatine $(\mathrm{PCr})$ resynthesis for energy supply during repeated short $(<10 \mathrm{~s})$ sprint sequences. For instance, the classic study of Gaitanos, Williams, Boobis and Brooks, (1993) showed an important contribution of the PCr metabolism for the regeneration of ATP in the last $\left(10^{\text {th }}\right)$ sprint of a very similar protocol $(10 \times$ 6s sprints with 30 s recovery), thus confirming that the participation of the glycolytic system decreases over the session together with an increase of the oxidative metabolism. These differences between the SIT5s and SIT20s could also be explained by the fact that PCr depletion depends on the duration of the sprint, being $\sim 45 \%$ of the basal value after 6s sprint, and $\sim 70 \%$ after a 20 s sprint (Billaut \& Bishop, 2009). Conversely, the longer the sprint, the greater the contribution of the glycolytic pathway (Balsom, Seger, Sjödin, \& Ekblom, 1992). Furthermore, $\mathrm{PCr}$ availability and resynthesis are crucially important factors for maintenance and recovery of power output (Bogdanis, Nevill, Boobis, \& Lakomy, 1996). In this regard, it has been proposed that PP is a critical determinant in the stimulation of physiological adaptations and that prolonged sprints are not required for further adaptations (Hazell, et al., 2010; Zelt, el al., 2014). More recently, Islam et al. (2017) observed that repetitions of running bouts of $15 \mathrm{~s}$ and $30 \mathrm{~s}$ elicited a greater decrease of peak speed ( $\sim 14$ and $\sim 27 \%$, respectively) when compared to $5 \mathrm{~s}$ bouts $(\sim 4 \%)$. These previous findings have been confirmed in the current study. Therefore, it could be suggested the appropriateness of very short sprints when looking for a greater PP output maintenance, a greater contribution of the aerobic metabolism, and hence a greater EE. This is an important aspect as the long-term adaptations after HIIT are related to the accumulated training load at higher intensities (Buchheit \& Laursen, 2013). Further longitudinal studies should verify if these different acute responses impact differently longterm adaptations of aerobic metabolism.

Previous studies reported significant correlations between $\mathrm{VO}_{2}$ and different mechanical parameters (Bishop, Edge, \& Goodman, 2004; Bishop \& Edge, 2006; Hamilton, Nevill, Brooks, \& Williams, 1991; Tomlin \& Wenger 2002). For instance, Bishop et al. $(2004,2006)$ reported moderate correlations $\left(\mathrm{r}=.60\right.$ and .64 ) beetween $\mathrm{VO}_{2}$ max and TW in untrained individuals and athletes, respectively. In line with this, we found positive correlations beetwen PP, MEP, TW and EE ( $\mathrm{r}=.62-.79)$, and negative correlations beetwen MEP, TW and meanRER $(r=-.69-.70)$, but only in SIT5s. This would reinforce the importance of the aerobic metabolism for a higher mechanical load, with a greater activity of the glycolytic pathway related to greater levels of fatigue and therefore a lower TW for the same exercise duration. The worsening in the capacity of force production in the myofibrillar proteins does appear largely attributable to a reduction of $\mathrm{Ca}^{2+}$ sensitivity as a result of acidosis (Cairns, 2006).

Previously, a significant correlation was reported between the changes in plasma $\left[\mathrm{H}^{+}\right]$and work $(\mathrm{r}=.41)$ and power decrement $(\mathrm{r}=.36)$ (Bishop, Edge, \& Goodman, 2004). Since the increment of $\mathrm{H}^{+}$ is directly associated with BLa increments (Cairns, 2006), it could be speculated that the greater BLa levels could be related to muscular fatigue. In the current study, the SIT20s showed a higher RER, $\triangle \mathrm{BLa}$ and RF. Therefore, given the different mechanical responses between the protocols despite having the same exercise duration, further studies should elaborate on the differences between SIT sessions when external loads are equated as both external and internal workload parameters seem to be related.

Contrary to our hypothesis and the recent findings of Townsend et al., (2017), we did not find significant differences between the protocols with respect to the perception of effort and affective responses (see Figure 3). Previously, it has been shown that a SIT session $(8 \times 30$ s at $130 \%$ of $\mathrm{P}_{\max }$ ) produced a higher perception of effort and a tendency for less positive affect than a HIIT session $\left(8 \times 60 \mathrm{~s}\right.$ at $85 \%$ of $\left.\mathrm{P}_{\max }\right)$ (Wood, et al., 2016). It has been also suggested that a greater activation of anaerobic metabolism could negatively influence feeling responses (Oliveira, Slama, 
Deslandes, Furtado, \& Santos, 2013). However, in the severe intensity domain, shorter efforts are more enjoyable (Martinez, Kilpatrick, Salomon, Jung, \& Little,2015). The recent work by Townsend et al. (2017) with running sprints observed that shorter efforts with increased repetitions produced more positive psychological responses. In this regard, it could be speculated that differences in exercise mode could explain these differences as our study was performed with a cycle ergometer. Previously, it has been reported that cycling promotes greater perception of effort than treadmill running for a given intensity (Green, et al., 2011). Additionally, it has been previously observed that training background can influence psychological responses during physical exertion (Garcin, et al., 2003). Given that the current sample was composed of active young individuals practicing different sports, the possible influence of this factor should not be excluded. Further studies should elaborate on the potential influence of these factors (exercise mode and training background) on psychological responses during different SIT protocols. Meanwhile, a greater efficiency of SIT5s could be suggested when looking for long-term adaptations, given that this protocol produced the higher mechanical and cardiorespiratory responses for the same psychological load.

One interesting finding was the significant differences detected in short-term HRR between the sessions (see Table 5). However, although both linear and non-linear HRV indices were depressed postexercise, no significant differences were observed between the sessions (see Table 4). Thus, SIT5s favored a faster parasympathetic reactivation immediately following the exercise (i.e. $\Delta 30$ s); however, the impact of both protocols on HRV indices was similar. To the best of our knowledge, this is the first study reporting differences in HRR after two SIT sessions. As the first 2 min of post-exercise recovery are mainly influenced by the parasympathetic reactivation (Stanley, et al., 2013), it could be suggested that the lower level of fatigue during SIT5s could be the main factor related to a faster parasympathetic reactivation after this protocol. On the other hand, previous studies examining the impact of different load indices on HRV after high-intensity exercises (Saboul, Balducci, Millet, Pialoux, \& Hautier, 2016; Stanley, et al., 2013), have observed a larger influence of exercise intensity when compared to other load parameters like duration or training volume (Saboul, et al., 2016). More recently, the study of Cipryan, Laursen, \& Plews (2016) showed a slight difference between efforts of $15 \mathrm{~s}$ vs. $30 \mathrm{~s}$ vs. $60 \mathrm{~s}$ work:relief HIIT sequences at the velocity associated with $\mathrm{VO}_{2} \max$, with the 30s:30s protocol showing the lower post-exercise HRV depression. As we did not observe such differences between the protocols, it could be speculated that this could be a consequence of the equated volume and workto-rest time between the sessions. Moreover, the expected influence of the metaboreflex activation on both HRR and HRV measures (Stanley, et al., 2013) has not been observed in the current study despite the higher glycolytic activity during SIT20s with the subsequent higher BLa after this protocol. Alternatively, we could speculate on the interaction of internal (e.g., lactate) and external (e.g., mechanical strain) factors on these responses. While we do not know the reason for this discrepancy, further studies should elaborate on the influence of SIT workloads, metaboreflex influences and training status on both HRR and HRV indices as these autonomic indices could present different sensitivity for identifying differences between the protocols.

The different effect of both SIT sessions on jump capacity reinforce the validity of $\mathrm{CMJ}$ as a simple test for assessing acute fatigue responses after high intensity efforts. Thus, in the current study, CMJ height was significantly lower only after the SIT20s (-4\%) (see Figure 4). Previous studies with repeated sprint sequences that evidenced almost perfectly correlations between jump height loss and BLa ( $\mathrm{r}=.96-.97)$, jump height loss and blood ammonia $(\mathrm{r}=.92-.95)$, and BLa and blood ammonia ( $\mathrm{r}=.94-.96)$ ( Jiménez-Reyes, et al., 2016; Morcillo, et al., 2015). Therefore, and in line with previous suggestions, it could be suggested that the greater glycolytic activity during SIT20s negatively affected explosive performance of the lower limbs. This has been confirmed in the current study with the significant correlation detected between $\triangle \mathrm{CMJ}$ and meanRER $(r=-.71 ; p=.014)$ only in SIT20s. Interestingly, when analyzing individual responses after both protocols, it is worth noting that some individuals potentiated their jump height after SIT5s (see Figure 4). This is an important observation as post-exercise CMJ height is the result of the balance between fatigue and potentiation (Boullosa, Abreu, Beltrame, \& Behm, 2013). From a practical point of view, this finding highlights the practicality of $\mathrm{CMJ}$ evaluation for assessing the readiness for performing strength exercises after endurance exercises of different intensities (Fyfe, et al., 2016).

It should be pointed out that the current SIT5s protocol could be considered an adapted version of repetitive sprint ability (RSA). In this regard, Taylor, Macpherson, Spears, \& Weston (2016) have recently suggested that RSA could be seen as a potent and time-efficient training strategy as it is effective in developing acceleration, speed, explosive leg power, aerobic power, and high-intensity-running performance. Thus, while the classic SIT protocols have been elaborated from a Wingate test (Gibala, et al., 2012), the recent suggestions of utilizing protocols with shorter sprints (Islam, et al., 2017; Townsend, et al., 2017; Vollaard \& Metcalfe, 2017), as in the current study, are also supported by the whole body 
of evidence that suggest RSA as an efficient and effective means for fitness development (Taylor, et al., 2016). This observation could be very important for public health policies. Further research, especially with sedentary and clinical populations, should elaborate on the number and duration of sprints for an optimal dose-response in every population, with special attention to individuals' adherence. Of note, the short duration of the sessions used in the current study (i.e., $\sim 12 \mathrm{~min}$ including warming up and cooling down) provides a reference for designing further protocols when looking for time-efficient interventions.

In summary, we have examined, for the first time, the physiological, perceptual and mechanical responses of different modified SIT sessions with different sprint duration but with the same exercise volume in healthy young males. The results of the current study suggest that a SIT protocol with very short sprints of $5 \mathrm{~s}$ favors greater mechanical workload and aerobic responses but with lower levels of fatigue. Therefore, the application of very short sprints may generate greater physiological adaptations. Further longitudinal studies should verify if these differences in acute responses between protocols are related to greater adaptations in the long term in different populations.

\section{Practical applications}

Given the current results, it is suggested the use of very short sprints during SIT sessions when looking for a higher mechanical strain and oxidative activity, but with lower fatigue. In contrast, when the objective is to tax the glycolitic pathway and promote higher levels of fatigue, longer sprints should be used. This information could be also important when designing complex training sessions in which aerobic training is performed before strength training. The use of simple monitoring tools as HRR and CMJ could be very usefull for detecting differences in fatigue levels after different SIT sessions.

\section{References}

Balsom, P.D., Gaitanos, G.C., Ekblom, B., \& Sjödin, B. (1994). Reduced oxygen availability during high intensity intermittent exercise impairs performance. Acta Physiologica, 152(3), 279-285. doi: 10.1111/j.1748-1716.1994. tb09807.x

Balsom, P.D., Seger, J.Y., Sjödin, B., \& Ekblom, B. (1992). Physiological responses to maximal intensity intermittent exercise. European Journal of Applied Physiology and Occupational Physiology, 65(2), 144-149.doi: 10.1007/ BF00705072

Batacan, R.B., Duncan, M.J., Dalbo, V.J., Tucker, P.S., \& Fenning, A.S. (2017). Effects of high-intensity interval training on cardiometabolic health: A systematic review and meta-analysis of intervention studies. British Journal of Sports Medicine, 51(6), 494-503.doi: 10.1136/bjsports-2015-095841

Billaut, F., \& Bishop, D. (2009). Muscle fatigue in males and females during multiple-sprint exercise. Sports Medicine, 39(4), 257-278.doi: 10.2165/00007256-200939040-00001

Billaut, F., \& Buchheit, M. (2013). Repeated sprint performance and vastus lateralis oxygenation: Effect of limited $\mathrm{O}_{2}$ availability. Scandinavian Journal of Medicine and Science in Sports, 23(3), e185-e193.doi: 10.1111/sms.12052

Bishop, D., \& Edge, J. (2006). Determinants of repeated-sprint ability in females matched for single-sprint performance. European Journal of Applied Physiology, 97(4), 373-379.doi: 10.1007/s00421-006-0182-0

Bishop, D., Edge, J., \& Goodman, C. (2004). Muscle buffer capacity and aerobic fitness are associated with repeatedsprint ability in women. European Journal of Applied Physiology, 92(4-5), 540-547.doi: 10.1007/s00421-0041150-1

Bogdanis, G.C., Nevill, M.E., Boobis, L.H., \& Lakomy, H.K. (1996). Contribution of phosphocreatine and aerobic metabolism to energy supply during repeated sprint exercise. Journal of Applied Physiology, 80(3), 876-884.

Boullosa, D.A., Abreu, L., Beltrame, L.G., \& Behm, D.G. (2013). The acute effect of different half squat set configurations on jump potentiation. Journal of Strength and Conditioning Research, 27(8), 2059-2066.doi: 10.1519/JSC.0b013e31827ddf15

Boullosa, D.A., Barros, E.S., Del Rosso, S., Nakamura, F.Y., \& Leicht, A.S. (2014). Reliability of heart rate measures during walking before and after running maximal efforts. International Journal of Sports Medicine, 35(12), 999-1005.doi: 10.1055/s-0034-1372637

Buchheit, M., \& Laursen, P.B. (2013). High-intensity interval training, solutions to the programming puzzle. Sports Medicine, 43(5), 313-338.doi: 10.1007/s40279-013-0029-x

Cairns, S.P. (2006). Lactic acid and exercise performance. Sports Medicine, 36(4), 279-291.doi: 10.2165/00007256200636040-00001

Cipryan, L., Laursen, P.B., \& Plews, D.J. (2016). Cardiac autonomic response following high-intensity running work-torest interval manipulation. European Journal of Sport Science, 16(7), 808-817.doi: 10.1080/17461391.2015.1103317 
Claudino, J.G., Cronin, J., Mezêncio, B., McMaster, D.T., McGuigan, M.,Tricoli, V.,Amadio A.C., \&Serrão J.C. (2016). The countermovement jump to monitor neuromuscular status: A meta-analysis.Journal of Science and Medicine in Sport, 20(4), 397-402.doi: 10.1016/j.jsams.2016.08.011

Dupont, G., Millet, G.P., Guinhouya, C., \& Berthoin, S. (2005). Relationship between oxygen uptake kinetics and performance in repeated running sprints. European Journal of Applied Physiology, 95(1), 27-34.doi: 10.1007/ s00421-005-1382-8

Fyfe, J.J., Bartlett, J.D., Hanson, E.D., Stepto, N.K., \& Bishop, D.J. (2016). Endurance training intensity does not mediate interference to maximal lower-body strength gain during short-term concurrent training. Frontiers in Physiology, 7,487.doi: 10.3389/fphys.2016.00487

Gaesser, G.A., \& Angadi, S.S. (2011). High-intensity interval training for health and fitness: Can less be more? Journal of Applied Physiology, 111,1540-1541.doi: 10.1152/japplphysiol.01237.2011.

Gaitanos, G.C., Williams, C., Boobis, L.H., \& Brooks, S. (1993). Human muscle metabolism during intermittent maximal exercise. Journal of Applied Physiology, 75(2), 712-719.

Garcin, M., Mille-Hamard, L., Devillers, S., Delattre, E., Dufour, S., \& Billat, V. (2003). Influence of the type of training sport practised on psychological and physiological parameters during exhausting endurance exercises. Perceptual and motor skills, 97(3), 1150-1162.10.2466/pms.2003.97.3f.1150

Gibala, M.J., \& Hawley, J.A. (2017). Sprinting toward fitness. Cell Metabolism, 25(5), 988-990.doi: 10.1016/j. cmet.2017.04.030

Gibala, M.J., Little, J.P., MacDonald, M.J., \& Hawley, J.A. (2012). Physiological adaptations to low volume, high intensity interval training in health and disease. Journal of Physiology, 590(5), 1077-1084.doi: 10.1113/jphysiol.2011.224725

Gillen, J.B., Percival, M.E., Skelly, L.E., Martin, B.J., Tan, R.B., Tarnopolsky, M.A., \&Gibala M.J. (2014). Three minutes of all-out intermittent exercise per week increases skeletal muscle oxidative capacity and improves cardiometabolic health. PLoS One, 9(11), el11489.doi: 10.1371/journal.pone.0111489

Green, J.M., Laurent, C.M., Bacon, N.T., ONeal, E.K., Davis, J.K., \& Bishop, P.A. (2011). Cross modal session rating of perceived exertion response at low and moderate intensities. Journal of Strength and Conditioning Research, 25(6), 1598-1604.doi: 10.1519/JSC.0b013e3181ddf6a2

Hamilton, A.L., Nevill M.E., Brooks, S., \&Williams, C. (1991). Physiological responses to maximal intermittent exercise: Differences between endurance-trained runners and games players. Journal of Sports Science,9, 371-382.

Hardcastle, S.J., Ray, H., Beale, L., \& Hagger, M.S. (2014). Why sprint interval training is inappropriate for a largely sedentary population?Frontiers in Psychology, 5, 1505. doi: 10.3389/fpsyg.2014.01505

Hardy, C.J., \& Rejeski, W.J. (1989). Not what, but how one feels: The measurement of affect during exercise. Journal of Sport and Exercise Psychology, 11(3), 304-317.

Hazell, T.J., MacPherson, R.E., Gravelle, B.M., \& Lemon, P.W. (2010). 10 or 30-s sprint interval training bouts enhance both aerobic and anaerobic performance. European Journal of Applied Physiology, 110(1), 153-160.doi: 10.1007/ s00421-010-1474-y

Islam, H., Townsend, L.K., \& Hazell, T.J. (2016). Modified sprint interval training protocols. Part I. Physiological responses. Applied Physiology, Nutrition, and Metabolism, 42(4), 339-346.doi: 10.1139/apnm-2016-0478

Jimenez-Reyes, P., Pareja-Blanco, F., Cuadrado-Peñafiel, V., Morcillo, J.A., Párraga, J.A., \& González-Badillo, J.J. (2016). Mechanical, metabolic and perceptual response during sprint training. International Journal of Sports Medicine, 37(10), 807-812.doi: 10.1055/s-0042-107251

Martinez, N., Kilpatrick, M.W., Salomon, K., Jung, M.E., \& Little, J.P. (2015). Affective and enjoyment responses to high-intensity interval training in overweight-to-obese and insufficiently active adults. Journal of Sport and Exercise Psychology, 37(2), 138-149.doi: 10.1123/jsep.2014-0212

McGawley,K., \&Bishop, D. (2015). Oxygen uptake during repeated-sprint exercise.Journal of Science and Medicine in Sport, 18, 214-218. doi: 10.1016/j.jsams.2014.02.002

Micklewright, D., Alkhatib, A., \& Beneke, R. (2006). Mechanically versus electro-magnetically braked cycle ergometer: Performance and energy cost of the Wingate anaerobic test. European Journal of Applied Physiology, 96(6), 748-751.doi: 10.1007/s00421-006-0145-5

Morcillo, J.A., Jiménez-Reyes, P., Cuadrado-Peñafiel, V., Lozano, E., Ortega-Becerra, M., \& Párraga, J. (2015). Relationships between repeated sprint ability, mechanical parameters, and blood metabolites in professional soccer players. Journal of Strength and Conditioning Research, 29(6), 1673-1682.doi: 10.1519/JSC.0000000000000782

Oliveira, B.R., Slama, F.A., Deslandes, A.C., Furtado, E.S., \& Santos, T.M. (2013). Continuous and high-intensity interval training: Which promotes higher pleasure?PLOSOne, 8(11), e79965.doi: 10.1371/journal.pone.0079965

Robertson, R.J., Goss, F.L., Dube, J., Rutkowski, J., Dupain, M., Brennan, C., \&Andreacci, J. (2004). Validation of the adult OMNI scale of perceived exertion for cycle ergometer exercise. Medicine and Science in Sports and Exercise, 36(1), 102-108.doi: 10.1249/01.MSS.0000106169.35222.8B

Saboul, D., Balducci, P., Millet, G., Pialoux, V., \& Hautier, C. (2016). A pilot study on quantification of training load: The use of HRV in training practice. European Journal of Sport Science, 16(2), 172-181.doi: 10.1080/17461391.2015.1004373

Skleryk, J.R., Karagounis, L.G., Hawley, J.A., Sharman, M.J., Laursen, P.B., \& Watson, G. (2013). Two weeks of reduced volume sprint interval or traditional exercise training does not improve metabolic functioning in sedentary obese men. Diabetes, Obesity and Metabolism, 15(12), 1146-1153.doi: 10.1111/dom.12150 
Sloth, M., Sloth, D., Overgaard, K., \& Dalgas, U. (2013). Effects of sprint interval training on $\mathrm{VO}_{2}$ max and aerobic exercise performance: A systematic review and meta analysis. Scandinavian Journal of Medicine and Science in Sports, 23(6), e341-e52.doi: 10.1111/sms.12092

Stanley, J., Peake, J.M., \& Buchheit, M. (2013). Cardiac parasympathetic reactivation following exercise: Implications for training prescription. Sports Medicine, 43(12), 1259-1277.doi: 10.1007/s40279-013-0083-4

Taylor, J.M., Macpherson, T.W., Spears, I.R, \& Weston, M. (2016). Repeated sprints: an independent not dependent variable. International Journal of Sports Physiology and Performance, 11(5), 693-696.doi: 10.1123/ijspp.20160081

Tomlin, D.L., \& Wenger, H.A. (2002). The relationships between aerobic fitness, power maintenance and oxygen consumption during intense intermittent exercise. Journal of Science and Medicine in Sport, 5(3), 194-203. doi: 10.1016/S1440-2440(02)80004-4

Townsend, L.K., Islam, H., Dunn, E., Eys, M., Robertson-Wilson, J., \& Hazell, T.J. (2017). Modified sprint interval training protocols. Part II. Psychological responses. Applied Physiology, Nutrition, and Metabolism, 42(4), 347-353.doi: 10.1139/apnm-2016-0479

Trost, S.G., Owen, N., Bauman, A.E., Sallis, J.F., \& Brown, W. (2002). Correlates of adults' participation in physical activity: Review and update. Medicine and Science in Sports and Exercise, 34(12), 1996-2001.doi: 10.1097/00005768-200212000-00020

Verney, J., Duclos, M., \& Thivel, D. (2017). Sprint interval training: What are the clinical implications and precautions?Medicine and Science in Sports and Exercise,49(11), 2361-2362.doi: 10.1249/MSS.0000000000001364

Vollaard, N.B., \& Metcalfe, R.S. (2017). Research into the health benefits of sprint interval training should focus on protocols with fewer and shorter sprints. Sports Medicine, 47(12), 2443-2451.doi: 10.1007/s40279-017-0727-x

Weippert, M., Behrens, K., Rieger, A., Kumar, M., \&Behrens, M. (2015). Effects of breathing patterns and light exercise on linear and nonlinear heart rate variability.Applied Physiology, Nutrition, and Metabolism, 40, 762-768.doi: 10.1139/apnm-2014-0493

Williams, C.B., Zelt, J.G., Castellani, L.N., Little, J.P., Jung, M.E., Wright, D.C.,Tschakovsky, M.E., \&Gurd, B.J.(2013). Changes in mechanisms proposed to mediate fat loss following an acute bout of high-intensity interval and endurance exercise. Applied Physiology, Nutrition, and Metabolism, 38(12), 1236-1244.doi: 10.1139/apnm2013-0101

Wood, K.M., Olive, B., LaValle, K., Thompson, H., Greer, K., \& Astorino, T.A. (2016). Dissimilar physiological and perceptual responses between sprint interval training and high-intensity interval training. Journal of Strength and Conditioning Research, 30(1), 244-250.doi: 10.1519/JSC.0000000000001042

Yamagishi, T., \& Babraj, J. (2017). Effects of reduced volume of sprint interval training and the time course of physiological and performance adaptations. Scandinavian Journal of Medicine and Science in Sports,27(12), 1662-1672.doi: 10.1111/sms.12831

Zelt, J.G., Hankinson, P.B., Foster, W.S., Williams, C.B., Reynolds, J., Garneys, E.,Tschakovsky, M.E., \&Gurd B.J. (2014). Reducing the volume of sprint interval training does not diminish maximal and submaximal performance gains in healthy men. European Journal of Applied Physiology, 114(11), 2427-2436.doi: 10.1007/s00421-014-2960-4

Submitted: October 28, 2017

Accepted: February 27, 2018

Published Online First: December 1, 2018

Correspondence to:

Daniel A. Boullosa, Ph.D.

Post-Graduate Program in Physical Education

Catholic University of Brasilia

QS 07, LT1 S/N -Room 111 - Block G, Águas Claras

71966-700

Brasilia, Brazil

E-mail: daniel.boullosa@gmail.com

Conflict of interests: The authors declare that they have no conflict of interest.

Acknowledgments: We would like to thank Danielle Garcia and Leticia Freire for their help during data collection.

Funding: Stefano Benítez-Flores is supported by a doctoral fellowship awarded by the ANII (Uruguay). Arilson Fernandes Mendonça de Sousa and Sebastian Del Rosso enjoy a PROSUP-CAPES grant. Thiago Santos Rosa enjoys a PNBD-CAPES grant. Daniel A. Boullosa enjoys a productivity research grant from CNPq (Brazil). 